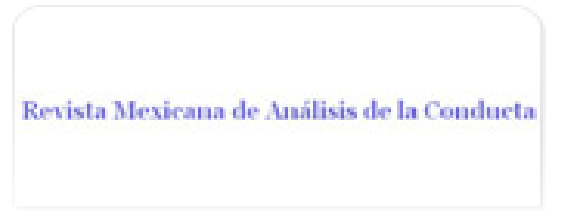

Revista Mexicana de Análisis de la Conducta ISSN: 0185-4534

editora@rmac-mx.org

Sociedad Mexicana de Análisis de la Conducta México

Calleja, Nazira; Hernández-Pozo, María del Rocío Prueba Stroop Computarizada de Riesgo Tabáquico para Adolescentes

Revista Mexicana de Análisis de la Conducta, vol. 35, núm. 2, diciembre, 2009, pp. 91-107

Sociedad Mexicana de Análisis de la Conducta

Guadalajara, México

Disponible en: http://www.redalyc.org/articulo.oa?id=59311902007

Cómo citar el artículo

- Número completo

- Más información del artículo

Página de la revista en redalyc.org

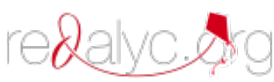

Sistema de Información Científica

Red de Revistas Científicas de América Latina, el Caribe, España y Portugal Proyecto académico sin fines de lucro, desarrollado bajo la iniciativa de acceso abierto 


\title{
Prueba Stroop Computarizada de Riesgo Tabáquico para Adolescentes
}

\author{
Stroop Test Automatized for Smoking \\ Risk Among Teenagers \\ NAZIRA CALLEJA Y MARÍA DEL ROCÍO HERNÁNDEZ-POZO' \\ UNIVERSIDAD NACIONAL AUTÓNOMA DE MÉXICO
}

\begin{abstract}
Resumen
Con el propósito de construir una prueba Stroop para evaluar la interferencia atencional producida por palabras vinculadas con fumar en adolescentes, se efectuaron dos estudios. En el primero se pidió a 473 estudiantes de secundaria, hombres y mujeres, que escribieran palabras positivas y negativas relacionadas con fumar o con tabaco. Con las 15 palabras positivas, las 15 negativas y las 15 asociadas con el tabaco de mayor frecuencia de aparición, y con sus correspondientes palabras neutras igualadas en longitud y frecuencia, se construyó una prueba Stroop de riesgo tabáquico computarizada, en tres versiones, las cuales variaban en el orden de presentación de los bloques. En el estudio 2 contestaron la prueba 164 adolescentes estudiantes de secundaria. Su tarea consistía en leer la palabra que aparecía en cada pantalla y digitar el número del color en el que estaba escrita. Se encontró que el orden de presentación de los bloques no afectó los índices de interferencia. Se obtuvieron correlaciones significativas del índice de interferencia ante palabras negativas con el de palabras asociadas con el tabaco, pero no con las positivas. Se concluyó que la prueba Stroop de riesgo tabáquico
\end{abstract}

1. Este estudio se realizó como parte de la investigación doctoral de la primera autora. La segunda autora contribuyó con el diseño del software y colaboró en el análisis y discusión de los hallazgos. Dirigir la correspondencia a las autoras Nazira Calleja, Facultad de Psicología, UNAM, México, ncalleja@camefe.com.mx y María del Rocío Hernández Pozo, Proyecto Aprendizaje Humano, División de Investigación y Posgrado, Edificio UIICSE, FES Iztacala, UNAM, herpoz@unam.mx.

Recibido: Septiembre 29, 2009. Revisado: Octubre 31, 2009. Aceptado: Noviembre 10, 2009. 
evalúan sesgos atencionales relacionados con fumar y con tabaco en los adolescentes.

Palabras clave: Prueba Stroop emocional, sesgo atencional, tabaco, fumar, tabaquismo, adolescentes

\begin{abstract}
A Stroop discriminative test was built to assess interference produced by words associated to tobacco use among teenagers. In the first phase of the study 473 junior high school students, men and women, wrote positive and negative words related to smoking or to tobacco, and neutral words not associated to the subject. Fifteen of the most frequent positive, negative and associated words were selected to create pairs with neutral words equal in length and frequency of use. Three versions of the computerized smoking risk Stroop test were built based upon selected dyads, by varying the order of the blocks. In the second phase of the study, 164 junior high school students, men and women, were exposed to the discriminative task, in which they had to press the number identifying the color in which the word was written on the screen. Order of the blocks did not affect interference indexes. Negative interferences correlated significantly with associated neutral words interference, but not with positive interference. It is concluded that the Stroop test for smoking risk assesses attentional bias related to smoking and to tobacco among adolescents, and that either order of block is equivalent for practical use.
\end{abstract}

Key words: Emotional Stroop test, attentional bias, tobacco, smoking, teenagers

Cuando se pide a una persona que responda a las características físicas de una palabra, como su color, y no a su significado, puede ocurrir un fenómeno de interferencia denominado "efecto Stroop", si y sólo si el significado de la palabra interfiere de alguna manera con la acción de nombrar su propiedad física. Este fenómeno se debe a que, a pesar de la instrucción, la persona procesa ambas dimensiones, lo que conduce a tiempos de reacción más lentos y a un mayor número de errores. John R. Stroop (1935) descubrió que las personas tienden a responder a estímulos verbales atendiendo, de manera preferente, a sus propiedades semánticas más que a las físicas. En su ya clásico experimento, Stroop (1935) pidió a los participantes que efectuaran dos tipos de tarea. En la primera, a la que denominó RCN (Reading Color Names), se les pedía que leyeran las palabras que nombraban el color en el que habían sido escritas; en la segunda (NCW, Naming Colored Words) debían identificar el color en el que las palabras estaban impresas, ignorando 
su contenido semántico. Cuando el color de la tinta era congruente con la palabra (e.g., la palabra "azul" escrita con tinta azul), el tiempo de identificación del nombre del color de la palabra se reducía; lo opuesto ocurría cuando no había congruencia (e.g., la palabra "rojo" escrita con tinta azul).

La mayoría de las explicaciones teóricas del efecto Stroop (MacLeod \& MacDonald, 2000) plantean que el tiempo que le toma a una persona leer las palabras es menor porque se trata de un proceso que ha sido activado con frecuencia en el pasado; en cambio, nombrar los colores requiere un esfuerzo de mayor atención.

Estudios de electroencefalografía, resonancia magnética y tomografía del efecto Stroop han revelado una activación selectiva de la circunvolución del cíngulo durante la realización de la tarea cuando se presenta la interferencia (Van den Heuvel et al., 2005). Esta estructura prefrontal se encuentra involucrada en una amplio rango de procesos de pensamiento y respuestas emocionales, y parece mediar la selección de una respuesta apropiada cuando se presentan señales en conflicto, lo cual ocurre durante la tarea Stroop (Bush, Whalen, Rosen, Jenike, Mcinerney \& Rauch, 1998).

La versión tradicional de la prueba Stroop (en español, Golden, 2005) consiste en la presentación de tres láminas con una secuencia de 100 respuestas cada una de ellas. En la primera lámina se solicita al sujeto que lea en voz alta palabras de colores ("rojo", "azul" y "verde", distribuidas al azar); en la segunda, se le pide que denomine el color de grupos de pequeñas aspas (rojo, azul y verde), y en la tercera, que denomine el color de la tinta en el que están impresas las palabras de colores, donde el color de la tinta y el color al que se refiere la palabra son incongruentes. En todas las láminas, se instruye al sujeto para que responda a la tarea tan rápido como pueda, pero sin cometer errores.

La prueba Stroop ha sido modificada para incluir otras modalidades sensoriales y otras variables. Se ha desarrollado, por ejemplo, una versión numérica de la tarea, denominada "Counting Stroop" (Bush et al., 1998). En ella se solicita a los participantes que indiquen el número de palabras (1 a 4) que aparecen en la pantalla, independientemente de su significado. Los ensayos de interferencia contienen un número de palabras que es incongruente con la respuesta correcta (e.g., la palabra "dos" escrita tres veces).

Existe también una versión automatizada del efecto de interferencia de Stroop (Richards \& Millwood, 1989). Las palabras se presentan una por una en el centro de la pantalla de la computadora escritas en mayúscula y con colores diferentes, y se solicita al sujeto una respuesta manual, no vocal, como en el caso de las láminas. La máquina contabiliza las latencias de respuesta, así como los aciertos y errores. Comúnmente, se emplean tres condiciones: una condición congruente (el nombre del color coloreado con su propio color); una condición neutral (una palabra neutra coloreada con 
alguno de los cuatro colores); y una condición incongruente (el nombre de un color coloreado con cualquiera de los otros tres colores (e.g., la palabra "azul" en color verde). Durante cada ensayo, a los participantes se les pide que indiquen el color en el que se encuentra escrita la palabra. La versión de computadora tiene la ventaja sobre la de láminas que permite al investigador examinar los tiempos de respuesta a cada uno de los estímulos y calcular automáticamente los índices de interferencia. La versión tradicional de la tarea de Stroop se ha empleado en investigación sobre adicciones en su versión computarizada, como una prueba conductual de impulsividad (Cuevas \& Hernández-Pozo, 2008).

Se ha desarrollado también una tarea emocional Stroop, en la que se presentan palabras con carga emocional para evaluar los sesgos atencionales asociados con ellas. La persona dirige su atención, más o menos automáticamente, hacia el significado de las palabras amenazantes, por lo que quedan se deteriora la ejecución para realizar la tarea principal, que es nombrar el color. Williams, Mathews \& MacLeod (1996) efectuaron una amplia revisión de estudios publicados utilizando la tarea Stroop y concluyeron que ésta ha probado su utilidad como una herramienta de evaluación clínica, que ofrece importantes oportunidades para avanzar en el entendimiento del sesgo atencional y la emoción. Se ha encontrado que el efecto de interferencia Stroop está presente en diversos trastornos clínicos como la depresión mayor (Gallardo, Baños, Belloch \& Ruipérez, 1999), el trastorno de pánico (Quero, Baños \& Botella, 2000), el dolor crónico (Anderson \& Haldrup, 2003) y la alexitimia (incapacidad de la persona para identificar y expresar verbalmente sus propias emociones; Martínez \& Marín, 1997).

De acuerdo con Torres, Hernández-Pozo, Castillo, Coronado \& Cerezo (2009), los índices de interferencia de la prueba emocional de Stroop, como la de ansiedad, se relacionan significativamente con medidas fisiológicas, como la presión sistólica.

La gran mayoría de las investigaciones han reportado su aplicación a poblaciones adultas; sólo algunos se han efectuado con niños o adolescentes. Por ejemplo, Martin, Holder \& Jones (1992) emplearon la prueba en niños de 6 a 13 años con palabras relacionadas con la fobia a las arañas; Moradi, Taghavi, Doost, Yule \& Dalgleish (1999) obtuvieron el efecto de interferencia en chicos de 9 a 17 años con estrés postraumático, y Macías \& HernándezPozo (2008) trabajaron con estudiantes de bachillerato, a quienes evaluaron en términos de su nivel de ansiedad escolar.

En diversos estudios se ha utilizado el paradigma emocional de Stroop para examinar los sesgos atencionales que ocurren en los fumadores respecto de palabras relacionadas con fumar (Drobes, Elibero \& Evans, 2006). Cox, Fadardi \& Pothos (2006) denominan a esta tarea "efecto Stroop-adicción"; en ese tipo de tarea los significados de las palabras se detectan más rápida- 
mente que los colores en los que están impresas, aun a pesar de que se de la instrucción explícita de que sean ignorados. La interferencia causada por los estímulos relacionados con la adicción se calcula obteniendo la diferencia entre la ejecución de los sujetos ante éstos y ante los estímulos neutros.

Aunque se han reportado algunos resultados inconsistentes (Kozuszek, 2008), en la mayoría de las investigaciones se ha encontrado que los fumadores, en relación con los no fumadores, presentan un mayor tiempo de reacción ante palabras relacionadas con fumar, que ante palabras neutras (Hernández-Pozo, 2009). Este efecto se presenta tanto en fumadores severos como en fumadores ligeros (Mogg, Field \& Bradley, 2005).

Zack, Belsito, Scher, Eissenberg \& Corrigall (2001) encontraron que la abstinencia de nicotina afectaba la ejecución de los participantes (hombres y mujeres de 14 a 18 años de edad) en la tarea Stroop clásica. Los fumadores severos ( $>11$ cigarros por día) mostraron más detrimento durante la privación y más mejoría después de fumar, en comparación con los fumadores ligeros, de manera que aún los fumadores noveles mejoran apreciablemente su habilidad para enfocarse en tareas intelectuales cuando fuman, un efecto que parece ser un incentivo para que continúen fumando.

El efecto emocional Stroop ha permitido evaluar también la relación entre el consumo de tabaco y los sesgos atencionales relacionados con las evaluaciones afectivas del fumador. Drobes et al. (2006) diseñaron una tarea Stroop con cuatro categorías de 16 palabras cada una: a) palabras relacionadas con fumar (e.g., "colilla", "nicotina", "cajetilla"), b) palabras afectivas negativas (e.g., "enfermo", "dolor", "culpa”), c) palabras afectivas positivas (e.g., "seguro", "contento", "esperanzado") y palabras neutras (e.g., "herramienta", "pala", "martillo"), igualadas en longitud, número de sílabas y frecuencia de ocurrencia en el idioma inglés. Los autores tomaron las palabras para su tarea de otros estudios o las crearon ellos mismos. También Gross, Murray \& Rosenblatt (1993), así como Waters \& Feyerabend (2000) construyeron de manera similar su tarea emocional Stroop. En México a la fecha, no se ha documentado la existencia de una prueba Stroop para estudiar la adicción al tabaco en adolescentes, por lo que el objetivo del presente estudio fue construir y aplicar una prueba Stroop de riesgo tabáquico en versión para computadora, dirigida específicamente a un grupo de la población: los adolescentes, ya que la prueba permitiría identificar a los individuos que se encuentran en riesgo de convertirse en adictos a la nicotina o que ya lo son, y emprender acciones preventivas o terapeúticas. Con el propósito de contar con una prueba válida y confiable, y para tener la seguridad de que los términos incluidos en la prueba pertenecieran al léxico de los propios adolescentes, la tarea se construyó con palabras generadas por ese grupo social. Se realizaron dos estudios: en el primero se construyó la prueba siguiendo el procedimiento indicado, y en segundo se aplicó y evaluó. 


\section{ESTUDIO 1. CONSTRUCCIÓN DE LA PRUEBA Método}

\section{Participantes}

473 estudiantes, hombres $(51.80 \%)$ y mujeres $(48.20 \%)$, de primero $(34.88 \%)$, segundo $(38.27 \%)$ y tercer $(26.85 \%)$ grados de una escuela secundaria pública $(23.68 \%)$ y cuatro privadas $(76.32 \%)$ de la zona sur de la ciudad de México. Sus edades oscilaron entre 11 y 16 años (media $=13.71)$. Fueron incluidos todos los estudiantes que asistieron a la escuela el día en que se efectuó la aplicación y que manifestaron su conformidad para participar.

\section{Procedimiento}

Se acudió a los salones de clase y se solicitó a los participantes que anotaran sus datos (escuela, edad, sexo y grado) en el formato que se les entregaba. No se les pidió su nombre. A continuación se les instruyó para que escribieran 10 palabras relacionadas con fumar o con tabaco que ellos consideraran positivas, 10 palabras relacionadas con fumar o con tabaco que ellos consideraran negativas y 10 palabras neutras que no tuvieran que ver con el tema de fumar o con tabaco.

Una vez recabadas las palabras, se procedió a su captura en una base de datos. Posteriormente, fueron agrupadas todas las palabras que compartían la misma raíz (por ejemplo: relaja, relajado, relajados, relajar, relajación, relajas, relajamiento, relajante) para cada bloque y se sumaron las frecuencias correspondientes a todas ellas. Se ordenaron en forma descendente de acuerdo con su frecuencia de aparición en cada tipo de palabras (positivas, negativas y neutras).

\section{Resultados}

Como se observa en la tabla 1, fueron generadas más palabras neutras que negativas, y más negativas que positivas. El porcentaje de palabras mencionadas una sola vez fue menor en las palabras positivas. Se encontró una mucho mayor concentración (i.e., palabras con frecuencias muy altas) entre las negativas y menor entre las neutras.

Las palabras neutras resultaron de una longitud promedio menor que las positivas y las negativas. 
Tabla 1

Número de palabras generadas por tipo

\begin{tabular}{|c|c|c|c|c|c|c|}
\hline & \multicolumn{2}{|c|}{ Negativas } & \multicolumn{2}{|c|}{ Positivas } & \multicolumn{2}{|c|}{ Neutras } \\
\hline Palabras diferentes & \multicolumn{2}{|c|}{408} & \multicolumn{2}{|c|}{385} & \multicolumn{2}{|r|}{471} \\
\hline Palabras con frecuencia $=1$ & & $4.90 \%)$ & & $9.09 \%)$ & & $(54.83 \%)$ \\
\hline \multirow{5}{*}{$\begin{array}{l}\text { Frecuencia de las cinco palabras } \\
\text { más altas }\end{array}$} & $1^{a}$ & 272 & $1^{a}$ & 148 & $1^{a}$ & 81 \\
\hline & $2^{\mathrm{a}}$ & 201 & $2^{a}$ & 95 & $2^{a}$ & 76 \\
\hline & $3^{a}$ & 144 & $3^{a}$ & 53 & $3^{a}$ & 70 \\
\hline & $4^{a}$ & 141 & $4^{a}$ & 53 & $4^{a}$ & 69 \\
\hline & $5^{a}$ & 112 & $5^{\mathrm{a}}$ & 44 & $5^{a}$ & 68 \\
\hline
\end{tabular}

Para construir la prueba Stroop de riesgo tabáquico se eligieron las 15 palabras positivas y las 15 negativas cuyas frecuencias de aparición fueron las más altas, siempre y cuando no aparecieran indistintamente en una u otra lista. Tal fue el caso de las palabras: nervios, amigos y rebelde. Las palabras seleccionadas fueron ordenadas de acuerdo con su longitud, definida por el número de letras, y para cada una de ellas se identificó, dentro de la lista de palabras neutras, una que fuera similar en frecuencia de aparición e igual en longitud. De la lista de palabras neutras se eliminaron aquéllas que discriminaban por género. Por ejemplo, las palabras futbol y balón fueron mucho más mencionadas por los hombres que por las mujeres y lo contrario ocurrió para las palabras amor y corazón.

Se identificaron asimismo 15 palabras asociadas con el tabaco, las cuales no tienen necesariamente una connotación positiva o negativa, como cerillos y ceniza. A cada una de ellas también se le hizo corresponder una palabra neutra de igual longitud.

Fueron seleccionadas nueve palabras neutras adicionales para los ensayos de práctica.

Las 15 díadas negativas-neutras y las 15 positivas-neutras, así como las palabras asociadas con tabaco y sus neutras, se muestran en la tabla 2. Para cada par se señala su longitud, el orden que corresponde a cada palabra de acuerdo con su frecuencia de aparición, así como el orden en el que se presentaron en la prueba. 
Tabla 2

Palabras que integran la prueba Stroop de riesgo tabáquico

\begin{tabular}{|c|c|c|c|c|}
\hline Díadas & Palabras & & $\begin{array}{c}\text { Longitud } \\
\text { (número de letras) }\end{array}$ & $\begin{array}{c}\text { Orden por frecuencia } \\
\text { de aparición }\end{array}$ \\
\hline \multicolumn{5}{|c|}{ Negativas-Neutras } \\
\hline 1 & enfisema & exámenes & 8 & $11^{\circ}$ \\
\hline 2 & vicio & silla & 5 & $13^{\circ}$ \\
\hline 3 & enfermedad & pantalones & 10 & $2^{\circ}$ \\
\hline 4 & cáncer & música & 6 & $1^{\circ}$ \\
\hline 5 & adicción & estudiar & 8 & $3^{\circ}$ \\
\hline 6 & pulmones & calcetín & 8 & $5^{\circ}$ \\
\hline 7 & gasto & carro & 5 & $12^{\circ}$ \\
\hline 8 & muerte & dulces & 6 & $4^{\circ}$ \\
\hline 9 & tos & sol & 3 & $10^{\circ}$ \\
\hline 10 & aliento & maestra & 7 & $7^{\circ}$ \\
\hline 11 & daño & gato & 4 & $6^{\circ}$ \\
\hline 12 & contamina & profesora & 9 & $8^{\circ}$ \\
\hline 13 & mata & hoja & 4 & $14^{\circ}$ \\
\hline 14 & malo & leer & 4 & $9^{\circ}$ \\
\hline 15 & garganta & pizarrón & 8 & $15^{\circ}$ \\
\hline \multicolumn{5}{|c|}{ Positivas- Neutras } \\
\hline 1 & relaja & zapato & 6 & $1^{\circ}$ \\
\hline 2 & placer & perros & 6 & $5^{\circ}$ \\
\hline 3 & moda & casa & 4 & $9^{\circ}$ \\
\hline 4 & tranquiliza & computadora & 11 & $2^{\circ}$ \\
\hline 5 & grande & comida & 6 & $6^{\circ}$ \\
\hline 6 & fiestas & celular & 7 & $11^{\circ}$ \\
\hline 7 & sabor & pluma & 6 & $7^{\circ}$ \\
\hline 8 & agradable & estrellas & 9 & $14^{\circ}$ \\
\hline 9 & desahogo & pulseras & 8 & $13^{\circ}$ \\
\hline 10 & ánimo & banca & 5 & $15^{\circ}$ \\
\hline 11 & atractiva & chamarras & 9 & $10^{\circ}$ \\
\hline 12 & calma & jugar & 5 & $12^{\circ}$ \\
\hline 13 & madurez & estuche & 7 & $8^{\circ}$ \\
\hline 14 & aceptada & ventanas & 8 & $3^{\circ}$ \\
\hline 15 & gusto & lápiz & 5 & $4^{\circ}$ \\
\hline \multicolumn{5}{|c|}{ Asociadas-Neutras } \\
\hline 1 & tabaco & animal & 6 & $3^{\circ}$ \\
\hline 2 & ceniza & puerta & 6 & $10^{\circ}$ \\
\hline 3 & colilla & familia & 7 & $12^{\circ}$ \\
\hline 4 & nicotina & plumones & 8 & $4^{\circ}$ \\
\hline 5 & tabaquismo & escritorio & 10 & $13^{\circ}$ \\
\hline 6 & humo & ropa & 4 & $2^{\circ}$ \\
\hline 7 & fumar & juego & 5 & $9^{\circ}$ \\
\hline 8 & marlboro & lámparas & 8 & $15^{\circ}$ \\
\hline 9 & olor & mesa & 4 & $1^{\circ}$ \\
\hline 10 & cajetilla & bicicleta & 9 & $5^{\circ}$ \\
\hline 11 & filtro & lentes & 6 & $7^{\circ}$ \\
\hline 12 & cenicero & convivir & 8 & $8^{\circ}$ \\
\hline 13 & lumbre & flores & 6 & $14^{\circ}$ \\
\hline 14 & cigarro & mochila & 7 & $6^{\circ}$ \\
\hline 15 & cerillos & hermanos & 8 & $11^{\circ}$ \\
\hline
\end{tabular}


Se construyeron tres versiones de la tarea que se diferenciaron por el orden de presentación de los bloques de los ensayos de las palabras positivas, negativas y asociadas. Cada una constó de 90 ensayos, más tres palabras neutras iniciales para los ensayos de práctica (véase tabla 3 ).

Tabla 3

Conformación de la prueba Stroop de Riesgo Tabáquico

\begin{tabular}{|c|c|c|c|c|c|}
\hline \multirow{2}{*}{$\begin{array}{l}\text { Versión Stroop Riesgo } \\
\text { Tabáquico }\end{array}$} & \multicolumn{5}{|c|}{ Orden de presentación de los bloques } \\
\hline & Ejemplos & $1^{\circ}$ & $2^{\circ}$ & $3^{\circ}$ & $4^{\circ}$ \\
\hline 1 & $\begin{array}{l}\text { Palabras } \\
\text { neutras de } \\
\text { práctica } \\
\text { (3) }\end{array}$ & $\begin{array}{l}\text { Palabras } \\
\text { neutras (45) }\end{array}$ & $\begin{array}{l}\text { Palabras } \\
\text { asociadas } \\
(15)\end{array}$ & $\begin{array}{l}\text { Palabras } \\
\text { positivas } \\
(15)\end{array}$ & $\begin{array}{l}\text { Palabras } \\
\text { negativas } \\
(15)\end{array}$ \\
\hline 2 & $\begin{array}{l}\text { Palabras } \\
\text { neutras de } \\
\text { práctica } \\
\text { (3) }\end{array}$ & $\begin{array}{l}\text { Palabras } \\
\text { neutras } \\
(45)\end{array}$ & $\begin{array}{l}\text { Palabras } \\
\text { negativas } \\
(15)\end{array}$ & $\begin{array}{l}\text { Palabras } \\
\text { asociadas } \\
(15)\end{array}$ & $\begin{array}{l}\text { Palabras } \\
\text { positivas } \\
(15)\end{array}$ \\
\hline 3 & $\begin{array}{l}\text { Palabras } \\
\text { neutras de } \\
\text { práctica (3) }\end{array}$ & $\begin{array}{l}\text { Palabras } \\
\text { neutras } \\
(45)\end{array}$ & $\begin{array}{l}\text { Palabras } \\
\text { positivas } \\
\text { (15) }\end{array}$ & $\begin{array}{l}\text { Palabras } \\
\text { negativas } \\
\text { (15) }\end{array}$ & $\begin{array}{l}\text { Palabras } \\
\text { asociadas } \\
\text { (15) }\end{array}$ \\
\hline
\end{tabular}

Las palabras neutras seleccionadas para los ensayos de práctica fueron para la versión 1: listones, paleta, bolsas; para la versión 2: mariposas, coche y corazón; y para la versión 3: bufanda, ojos, sacapuntas.

Con las palabras obtenidas se elaboró un programa de computadora que permitía presentar las palabras por bloques (balanceando los colores de la palabra muestra, la posición de las opciones en el menú de elección y las respuestas correctas), recabar las respuestas y calcular los índices de interferencia de manera automática.

\section{Descripción de la prueba Stroop de riesgo tabáquico}

En cada ensayo se presenta una palabra con carga emocional negativa, positiva o asociada con el tabaco, o una palabra neutra no relacionada con el tema. Cada palabra aparece en la parte superior de la pantalla, escrita con letras mayúsculas, y con fondo negro. Las palabras se presentan coloreadas aleatoriamente en uno de seis colores: rojo, amarillo, morado, verde, gris y azul; a cada color corresponde un número. A los cuatro colores comúnmente empleados en la tarea Stroop, se agregaron dos más a fin de disminuir la probabilidad de responder correctamente por azar $(p=0.250$ versus $p=0.166)$.

La tarea del participante consiste en leer en voz baja la palabra e identificar, lo más rápido posible y sin cometer errores, el color en el que apa- 
rece escrita la palabra, seleccionarlo a partir del menú de colores que se encuentra debajo de la palabra y cuyo arreglo cambia de un ensayo a otro, con 30 arreglos diferentes, y digitar en el teclado horizontal el número que corresponde a tal color. Después de presionar la tecla aparece la siguiente palabra, y así sucesivamente hasta concluir los 90 ensayos. Las primeras pantallas proporcionan las instrucciones detalladamente y muestran, en pantallas sucesivas, el nombre del color en su color correspondiente para que el participante pueda identificarlo fácilmente. Los tres primeros ensayos son de prueba para familiarizarlo con la tarea. Un ejemplo de las pantallas utilizadas se presenta en la figura 1.

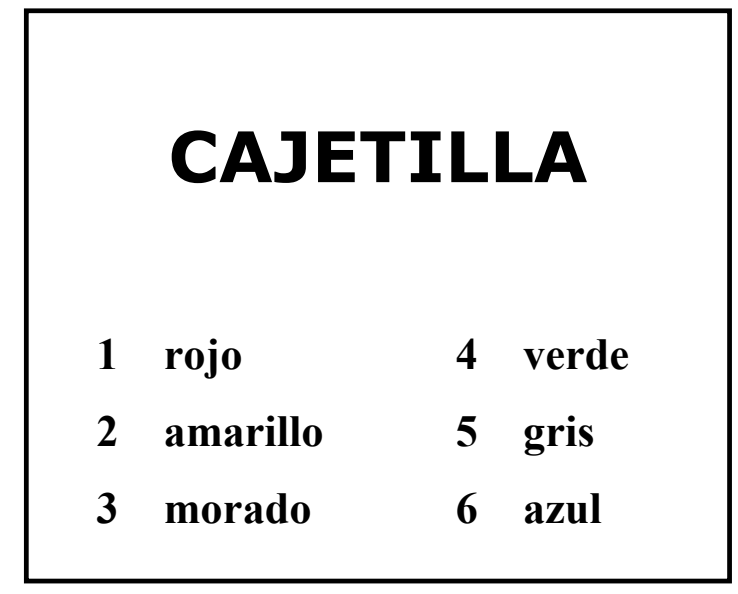

Figura 1. Pantalla de la prueba Stroop de riesgo tabáquico.

En la pantalla, el fondo aparecía en negro; la palabra muestra, en este caso "cajetilla", se presentaba en color morado, y el menú de seis opciones con nombres de colores, en blanco. Para la pantalla de la figura 1, la respuesta correcta es el número 6, correspondiente a la palabra "morado", que coincide con el color en que se encuentra escrita la palabra muestra.

El programa genera un archivo para cada participante en el que se registran los aciertos obtenidos, la latencia de respuesta ante cada palabra (tiempo transcurrido, en milisegundos, entre la presentación de la palabra estímulo y la respuesta), así como los índices de interferencia.

El cálculo de los índices de interferencia se realiza mediante la resta de las latencias ante las palabras neutras a las latencias ante las palabras negativas, positivas y asociadas de los bloques tabáquicos de la misma díada 
de palabras, igualadas en longitud y en probabilidad lexical. De ese modo se obtienen los índices negativos, positivos y asociados, respectivamente.

En los casos en los que los puntajes de interferencia resultan negativos, es decir, cuando las latencias ante las palabras neutras son superiores a las latencias ante las palabras negativas, positivas o asociadas del tabaco, ocurre una ausencia de sesgo atencional (el participante no se tarda más en identificar el color en que estaba escrita una palabra tabáquica que una neutra), por lo que los valores se convierten en cero.

Respecto de los aciertos, definidos como el número de ensayos en los que el participante indica correctamente el color en el que se encuentra escrita la palabra muestra, se estableció un criterio mínimo de ejecución (80 de 90 aciertos); en caso de no alcanzar los aciertos mínimos en una sesión, el participante tenía que repetir la prueba.

\section{ESTUDIO 2. APLICACIÓN DE LA PRUEBA Método}

\section{Participantes}

164 estudiantes, 80 hombres y 84 mujeres, de 11 a 15 años de edad, de $1^{\circ}$ $(34.2 \%), 2^{\circ}(32.9 \%)$ y $3^{\text {er }}(32.9 \%)$ grados de dos escuelas secundarias, una pública $(61.0 \%$ ) y una privada $(39.0 \%)$, de la zona sur de la ciudad de México.

\section{Aparatos y materiales}

El programa de la prueba Stroop de riesgo tabáquico fue instalado en ocho computadoras, cuatro por escuela, con procesador Celeron o Pentium, 512 MB de memoria y disco duro de $80 \mathrm{~GB}$, con lector de disco de $3.5 \mathrm{y} / \mathrm{o}$ unidad de CD y sistema operativo Windows 98.

\section{Procedimiento}

Después de solicitar a las autoridades escolares el acceso, se instaló el programa en cuatro de las computadoras del salón de cómputo de cada una de las escuelas, en las cuales el programa funcionaba adecuadamente y podía recuperarse el archivo de respuestas. Por tanto, se trabajó de manera simultánea con un máximo de cuatro estudiantes.

Se efectuó un estudio piloto de la prueba con 10 estudiantes. Como consecuencia de esta aplicación, se realizaron ajustes en las pantallas de instrucciones, a fin de facilitar el entendimiento de la tarea. 
Los participantes eran conducidos de su salón de clases al de cómputo. Una vez que se sentaban frente a la computadora, el aplicador se aseguraba que introdujeran correctamente el código que se les proporcionaba para identificar el archivo, leía con ellos las pantallas de instrucciones y resolvía las dudas que surgieran.

Al concluir los 90 ensayos, aparecía, en la pantalla final, el número de aciertos que había obtenido el participante. Si era menor de 80 , cifra establecida como criterio mínimo de ejecución, se le pedía que volviera a hacer la prueba. Para asegurar que el aplicador pudiera constatar el número de aciertos logrados, sólo se podía salir de la última pantalla con una clave especial que los participantes desconocían.

La versión de la prueba con la que trabajaron los estudiantes se asignó aleatoriamente. Al $27.4 \%$ le correspondió la versión 1, al $37.2 \%$, la 2 y al $35.4 \%$, la 3 .

\section{Resultados}

El programa de la prueba generó un archivo de respuestas para cada participante. Los índices de interferencia, obtenidos a partir de las latencias de respuesta, fueron transferidos y procesados con el paquete SPSS versión 12.0.

Con el propósito de determinar si el orden de presentación de los bloques por tipo de palabras (negativas, positivas y asociadas) afectaba los índices de interferencia, se compararon los grupos que habían respondido a las diferentes versiones. No se encontraron diferencias entre las versiones en ninguno de los índices.

La mayoría de los participantes $(92.2 \%)$ respondieron correctamente por lo menos 86 de los 90 ensayos (media $=88.3$ ).

Cerca de dos terceras partes de los integrantes de la muestra presentaron interferencia ante los tres tipos de palabras (negativas: $73.2 \%$; positivas: $70.7 \%$; asociadas: $71.3 \%$ ). Las medias y las desviaciones estándar de cada uno de los índices para los participantes con interferencia se muestran en la tabla 4. 
Tabla 4

Estadísticos de índices de interferencia de riesgo tabáquico

\begin{tabular}{lccc}
\hline Estadísticos & $\begin{array}{c}\text { Ante palabras } \\
\text { negativas }\end{array}$ & $\begin{array}{c}\text { Ante palabras } \\
\text { positivas }\end{array}$ & $\begin{array}{c}\text { Ante palabras } \\
\text { asociadas }\end{array}$ \\
\hline Media & 485.28 & 485.97 & 533.55 \\
Desviación estándar & 322.18 & 343.67 & 438.13 \\
Rango & $1-1289$ & $4-1440$ & $3-2091$ \\
\hline
\end{tabular}

El índice de interferencia ante palabras asociadas correlacionó significativamente con el índice de interferencia ante palabras negativas $(r=.399$, $p=.000$ ), pero no ante palabras positivas; la correlación de los índices ante palabras negativas y positivas no fue significativa.

Para quienes presentaron interferencia, se probaron diferencias por sexo, grado y tipo de escuela en los tres índices. Sólo en el índice ante palabras asociadas con el tabaco las mujeres (media $=621.54$; desviación estándar $=506.41$ ) difirieron de los hombres (media $=437.70$; desviación estándar $=$ 327.35); $t(115)=2.309, p=.023$. En el índice de interferencia ante palabras negativas, los participantes que asistían a una escuela privada obtuvieron mayores puntajes $($ media $=534.89$; desviación estándar $=320.74)$, en comparación con los estudiantes de escuela pública (media: 410.88; desviación estándar $=313.02) ; \mathrm{t}(118)=2.095, \mathrm{p}=.038$. No hubo diferencias por grado escolar en ninguno de los índices.

\section{DISCUSIÓN}

La prueba Stroop comúnmente se ha aplicado a niños y adolescentes bajo el paradigma tradicional, el cual consta de tres partes: en la primera se leen las palabras rojo, verde y azul escritas en negro sobre fondo blanco; en la segunda -denominada de colores- se nombra el color rojo, verde o azul de pequeñas aspas, y en la tercera -de color-palabras- aparecen las palabras rojo, verde y azul impresas en un color de tinta incongruente (Félix, 2006). Se ha utilizado, por ejemplo, para evaluar funciones atentivas en chicos con Trastorno de Déficit de Atención e Hiperactividad, TDAH (Dos Santos Assef, Capovilla \& Capovilla, 2007). Sin embargo, a la fecha no existen datos publicados del uso de la versión emocional del paradigma de Stroop en chicos mexicanos. Durante la aplicación de la prueba Stroop de riesgo tabáquico se observó que ésta resultó de interés y de respuesta sencilla para los participantes, quienes la respondieron mayoritariamente sin errores, a pesar de que se emplearon seis colores en lugar de los cuatro usuales (Cox et al., 2006, afirman que mientras mayor es el número de alternativas de respuesta 
en la tarea, ocurre mayor interferencia ya que se requiere mayor esfuerzo cognitivo para elegir la respuesta correcta), por lo que podría utilizarse en poblaciones similares para el estudio de diversos fenómenos de interés clínico y psicosocial.

Respecto de la generación de las palabras que permitieron la construcción de la prueba Stroop de riesgo tabáquico, cabe destacar la emisión de un mayor número y dispersión de palabras neutras que de palabras relacionadas con el tabaco, además de que su longitud promedio fue menor. Entre las neutras surgieron principalmente prendas de vestir y accesorios, así como artículos escolares y de papelería. La frecuencia de algunas palabras apuntó los intereses diferenciales de las niñas y los niños, de lo cual se deriva que este tipo de prueba, basada en la versión emocional del paradigma de Stroop, podría ser de utilidad para evaluar confiablemente variables como masculinidad-feminidad, al incluir palabras con mayor frecuencia de uso por uno $u$ otro género.

Se registraron más palabras negativas que positivas relativas al taba$c o, y$ sus frecuencias fueron altas, lo cual lleva a considerar que, para los adolescentes, palabras como cáncer, enfermedad, adicción, muerte y daño se encuentran estrechamente vinculadas con fumar, y que, como ya se ha señalado, en la adicción al tabaco intervienen factores adicionales a la información sobre sus efectos dañinos (Rooney \& Villahoz-González, 1994).

En relación con las palabras positivas asociadas con tabaco o fumar, la mayoría de las palabras más mencionadas tienen que ver con los efectos químicos de la nicotina: relaja, tranquiliza, gusto, placer, calma, desahogo, ánimo, o bien, con sus beneficios sociales: aceptada, grande, madurez, moda, atractiva, fiestas. De lo anterior resulta claro que los adolescentes conocen las propiedades relajantes y estimulantes de la nicotina, así como la imagen social del(a) fumador(a) que la publicidad ha vinculado con el tabaco y a la que ellos(as) aspiran (Koh, Joossens \& Connolly, 2007).

Si bien originalmente se planteó incluir sólo palabras negativas y positivas relacionadas con fumar o con el tabaco, al recabar las palabras para la construcción de la prueba surgieron términos que no tenían una connotación clara, ya que fueron mencionados por algunos participantes como negativas y por otros como positivas. Tal es el caso de palabras como filtro, cerillos, cajetilla, lumbre, cigarro y cenicero, que si bien se encuentran vinculadas con fumar y con tabaco, no hablan directamente de sus beneficios o daños. Se decidió, por tanto, crear un bloque más de palabras a las que se denominó "asociadas". Los índices de interferencia ante estas palabras y su rango fueron mayores que ante las negativas o las positivas, lo cual muestra la mayor dispersión que éstas generan. Un estudio cualitativo de las palabras asociadas permitiría acercarse al significado que cada una de ellas tiene para los adolescentes. 
Este tipo de palabras, denominadas aquí asociadas con el tabaco, han sido utilizadas en investigaciones que emplean la tarea emocional Stroop para estudiar la adicción al tabaco (e.g., Gross et al., 1993; Waters \& Feyerabend, 2000). Las palabras colilla, humo, filtro, cajetilla, cerillos, tabaco, nicotina y cigarro empleadas aquí, fueron aplicadas también por Drobes et al. (2006), en inglés; estos autores incluyeron otras, como alquitrán, mentolados, inhalar, bocanada, que no estuvieron en el presente estudio, y en éste se presentaron tabaquismo, marlboro, lumbre y olor, no incluidos por ellos.

Al inicio del estudio, se hipotetizó que el orden de presentación de los bloques de palabras negativas, positivas y asociadas con el tabaco afectaría los índices de interferencia. Los resultados no mostraron ninguna diferencia entre los índices de los grupos que recibieron los diferentes ordenamientos. Por tanto, puede afirmarse que es posible usar indistintamente cualquiera de las tres versiones, para efecto de la medición del sesgo atencional ante el tema de fumar o consumir tabaco.

Se observó una correlación positiva y significativa entre los índices de interferencia ante palabras asociadas y negativas; en cambio, la interferencia ante palabras positivas no correlacionó con ninguno de los otros dos grupos de palabras. Estos resultados apoyan los hallazgos de Drobes et al. (2006), quienes encontraron que, entre fumadores, las palabras relacionadas con fumar (e.g., cigarro, fumada, humo) correlacionaron significativamente con las de afecto negativo (e.g, enfermo, dolor, culpa), pero no con las de afecto positivo (e.g., seguro, contento, esperanzado).

Se encontraron diferencias estadísticamente significativas en el índice de interferencia ante palabras asociadas por sexo y en el de palabras negativas por tipo de escuela. Futuros estudios deberán explorar los factores relacionados con el hecho de que las mujeres vs. los hombres y los estudiantes de escuelas públicas vs. de los de escuelas privadas, obtengan mayores puntajes en tales índices.

Una prueba emocional de Stroop en español diseñada para población adulta, con palabras relacionadas con fumar y palabras neutras, que empleó también seis colores como opciones de selección en 60 ensayos, arrojó valores medios de interferencia negativa de 207.1 para fumadores, contra 170 para no fumadores, (Hernández-Pozo, 2009), siendo el rango de la distribución de índices de interferencia negativa inferior al encontrados con adolescentes.

La prueba emocional Stroop de riesgo tabáquico construida para adolescentes permitió detectar sesgos atencionales asociados con fumar y con tabaco; sin embargo, se requiere aún estudiar la relación existente entre los índices de interferencia ante los distintos tipos de palabras (negativas, positivas y asociadas) y el comportamiento tabáquico de los adolescentes, a fin de validar la prueba para ese grupo de edad, e identificar mediante ese 
procedimiento los sesgos de los jóvenes que requieren ser incorporados a programas preventivos o a tratamientos de cesación de tabaquismo.

\section{REFERENCIAS}

Anderson, G., \& Haldrup, D. (2003). Personalized pain words and Stroop interference in chronic pain patients. European Journal of Pain, 7, 431-438.

Bush, G., Whalen, P. J., Rosen, B. R., Jenike, M. A., Mcinerney, S. C., \& Rauch, S. L., (1998). The counting Stroop: An interference task specialized for functional neuroimaging: Validation study with functional MRI. Human Brain Mapping, 6, 270-282.

Cox, W. M., Fadardi, J. S., \& Pothos, E. M. (2006). The Addiction-Stroop Test: Theoretical considerations and procedural recommendations. Psychological Bulletin, 132, 443-476.

Cuevas, E., \& Hernández-Pozo, M. R. (2008). Evaluación de la impulsividad en fumadores: Una revisión sistemática. Revista Mexicana de Análisis de la Conducta, 34, 293-311.

Dos Santos Assef, E. C., Capovilla, A. G. S., \& Capovilla, F. C. (2007). Computerized Stroop test to assess selective attention in children with Attention Deficit Hyperactivity Disorder. The Spanish Journal of Psychology, 10, 33-40.

Drobes, D., Elibero, A., \& Evans, D. E. (2006). Attentional bias for smoking and affective stimuli: A Stroop task study. Psychology of Addictive Behaviors, 20, 490-495.

Félix, M. V. (2006). Recursos para el diagnóstico psicopedagógico del TDAH y comorbilidades. Revista Electrónica de Investigación Psicoeducativa, 10, 623-642.

Gallardo, M., Baños, R. M., Belloch, A., \& Rupierez, M. A. (1999). Sesgo atencional y vulnerabilidad de la depresión. The Spanish Journal of Psychology, 2, 11-19.

Golden, C. J. (2005). Test de colores y palabras (Stroop). Madrid: TEA.

Gross, T. M., Murray, J. E., \& Rosenblatt, M. R. (1993). Nicotine abstinence produces content-specific Stroop interference. Psychopharmacology, 111, 333-336.

Hernández-Pozo, M. R. (2009) Prejuicio selectivo a palabras relacionadas con tabaco en fumadores y no fumadores. Manuscrito enviado a publicación.

Koh, H. K., Joossens, L. X., \& Connolly, G. N. (2007). Making smoking history worldwide. The New England Journal of Medicine, 356, 1496-1498.

Kozuszek, W. F. (2008). The Stroop task and attentional bias in smokers. Thesis. Master Degree of Arts in Psychology. Humboldt State University, Arcata, CA, USA.

Macías, D., \& Hernández-Pozo, M. R. (2008). Indicadores conductuales de ansiedad escolar en bachilleres en función de sus calificaciones en un examen de Matemáticas. Revista Universitas Psychologica, 7, 767-785.

MacLeod, C. M., \& MacDonald, P. A. (2000). Interdimensional interference in the Stroop effect: Uncovering the cognitive and neural anatomy of attention. Trends in Cognitive Science, 4, 383-391.

Martin, M., Holder, P., \& Jones, G. V. (1992). Integral bias in naming of phobia-related words. Cognition and Emotion, 6, 479-484. 
Martínez, F., \& Marín, J. (1997). Influencia del nivel de alexitimia en el procesamiento de estímulos emocionales en una tarea Stroop. Psicothema, 9, 519-527.

Mogg, K., Field, M., \& Bradley, B. P. (2005). Attentional and approach biases for smoking cues in smokers: An investigation of competing theoretical views of addiction. Psychopharmacology, 180, 333-341.

Moradi, A. R., Taghavi, M. R., Doost, H. T. N., Yule, W., \& Dalgleish, T. (1999). Performance of children and adolescents with PTSD on the Stroop color-naming task. Psychological Medicine, 29, 415-419.

Quero, S., Baños, R. M., \& Botella, C. (2000). Sesgos cognitivos ene I trastorno de pánico: comparación entre el Stroop computarizada y con tarjetas. Psicothema, 12, 165-170.

Richards, A., \& Millwood, B. (1989). Colour-identification of diferentially valenced words in anxiety. Cognition and Emotion, 3, 171-176.

Rooney, J. F., \& Villahoz-González, J. (1994). Variables que contribuyen a fumar tabaco entre una muestra joven: Un análisis multivariable. Revista de Psicología Social, 9, 165-178.

Stroop, J. R. (1935). Studies of interference in serial verbal reactions. Journal of Experimental Psychology, 12, 643-662.

Torres, M., Hernández-Pozo, M. R., Castillo, P., Coronado, O., \& Cerezo, S. (2009). Relación entre promedio escolar y tres indicadores de ansiedad general en estudiantes de Medicina. Revista Universitas Psychologica, 8, 69-86.

Van den Heuvel, O. A., Veltman, D. J., Groenewegen, H. J., Witter, M. P., Merkelbach, J., Cath, D. C., et al. (2005). Disorder-specific neuroanatomical correlates of attentional bias in obsessive-compulsive disorder, panic disorder, and hypochondriasis. Archives of General Psychiatry, 62, 922-933.

Waters, A. J., \& Feyerabend, C. (2000). Determinants and effects of attentional bias in smokers. Psychology of Addictive Behaviors, 14, 111-120.

Williams, J. M. G., Mathews, A., \& MacLeod, C. (1996). The Emotional Stroop Task and Psychopathology. Psychological Bulletin, 120, 3-24.

Zack, M., Belsito, L., Scher, R., Eissenberg, T., \& Corrigall, W. A. (2001). Effects of abstinence and smoking on information processing in adolescent smokers. Psychopharmacology, 153, 249-257. 